\title{
L-Carnitine counteracts in vitro fructose-induced hepatic steatosis through targeting oxidative stress markers
}

\author{
A. Montesano ${ }^{1} \cdot$ P. Senesi ${ }^{1} \cdot$ F. Vacante ${ }^{2} \cdot$ G. Mollica ${ }^{1,2} \cdot$ S. Benedini ${ }^{1} \cdot$ M. Mariotti $i^{3,4} \cdot$ L. Luzi $^{1,2} \cdot$ I. Terruzzi $^{1,2}$
}

Received: 1 April 2019 / Accepted: 21 October 2019 / Published online: 8 November 2019

(c) The Author(s) 2019

\begin{abstract}
Purpose Nonalcoholic fatty liver disease (NAFLD) is defined by excessive lipid accumulation in the liver and involves an ample spectrum of liver diseases, ranging from simple uncomplicated steatosis to cirrhosis and hepatocellular carcinoma. Accumulating evidence demonstrates that high fructose intake enhances NAFLD development and progression promoting inhibition of mitochondrial $\beta$-oxidation of long-chain fatty acids and oxidative damages. L-Carnitine (LC), involved in $\beta$-oxidation, has been used to reduce obesity caused by high-fat diet, which is beneficial to ameliorating fatty liver diseases. Moreover, in the recent years, various studies have established LC anti-oxidative proprieties. The objective of this study was to elucidate primarily the underlying anti-oxidative mechanisms of LC in an in vitro model of fructose-induced liver steatosis. Methods Human hepatoma HepG2 cells were maintained in medium supplemented with LC (5 mM LC) with or without $5 \mathrm{mM}$ fructose (F) for $48 \mathrm{~h}$ and $72 \mathrm{~h}$. In control cells, LC or F was not added to medium. Fat deposition, anti-oxidative, and mitochondrial homeostasis were investigated.

Results LC supplementation decreased the intracellular lipid deposition enhancing AMPK activation. However, compound $\mathrm{C}$ (AMPK inhibitor-10 $\mu \mathrm{M}$ ), significantly abolished LC benefits in F condition. Moreover, LC, increasing PGC1 $\alpha$ expression, ameliorates mitochondrial damage-F induced. Above all, LC reduced ROS production and simultaneously increased protein content of antioxidant factors, SOD2 and Nrf2.

Conclusion Our data seemed to show that LC attenuate fructose-mediated lipid accumulation through AMPK activation. Moreover, LC counteracts mitochondrial damages and reactive oxygen species production restoring antioxidant cellular machine. These findings provide new insights into LC role as an AMPK activator and anti-oxidative molecule in NAFLD.
\end{abstract}

Keywords Hepatic steatosis $\cdot$ Metabolic disease $\cdot$ Fructose $\cdot$ L-Carnitine $\cdot$ Lipid deposition $\cdot$ Oxidative stress

A. Montesano, P. Senesi and F. Vacante contributed equally to this work.

\section{Terruzzi}

Ileana.terruzzi@unimi.it

1 Department of Biomedical Sciences for Health, University of Milan, Milan, Italy

2 Metabolism Research Center, IRCCS Policlinico San Donato, San Donato Milanese, Milan, Italy

3 IRCCS Istituto Ortopedico Galeazzi, Milan, Italy

4 Department of Biomedical, Surgical and Dental Sciences, University of Milan, Milan, Italy

\section{Introduction}

Nonalcoholic fatty liver disease (NAFLD), one of the most common cause of chronic liver diseases, is characterized by the abundant accumulation of triglycerides in hepatocytes, a condition that starts from a simple steatosis and may further progress to steatohepatitis (NASH), cirrhosis, and hepatocellular carcinoma [1,2]. Since NAFLD patients are often overweight, over-nutrition achieves a fundamental role during the pathogenesis of hepatic lipid accumulation [3]. Several investigations have demonstrated that a fructose $(\mathrm{F})$ overconsumption is involved in NAFLD progression, stimulating de novo lipogenesis, production and secretion of triglyceride and very lowdensity lipoprotein, and blocking fatty acid oxidation [4]. Moreover, recent data suggest how a chronically high fructose intake could inhibit AMP-activated protein kinase 
(AMPK), the main energy sensor of cellular metabolism, whereas its activation counteracts NAFLD progression [5]. Additionally, literature documents have indicated that fructose-rich diet is associated with oxidative stress and, in particular, with the decrease of mitochondrial biogenesis and antioxidant machine [6]. In particular, high fructose intake leads to a dysregulation of nuclear factor E2-related factor 2 (Nrf2) expression that regulates mitochondrial antioxidant function enhancing synthesis of detoxifying enzymes [7]. Recently, Sharma et al. have revealed how in mice fed high fat plus fructose, Nrf2 pharmacological activation ameliorates insulin resistance and alleviates NASH and liver fibrosis, principally decreasing oxidative stress and inflammatory [8].

If an imbalance diet and altered cellular metabolism are the main causes of NAFLD progression, eating habit modifications and, in general, weight reduction remain a firstline strategy in NAFLD management [9]. The nutritional recommendations are even more important considering that a specific pharmacological intervention for NAFLD has not yet been identified. In effect, the various pharmacological treatments use specific drugs for coexisting diseases, namely the glucagon-like peptide 1 and the cotransport antagonist 2 sodium/glucose (SGLT-2) for the control of glycaemia, in association with vitamin E supplementation [10]. Recently, novel therapeutic options for NAFLD have been proposed including activation of farnesoid $\mathrm{X}$ receptor (FXR) that ameliorates fibrotic and inflammatory damages [11, 12]. However, Mediterranean diet prefers low glycemic index products and antioxidant foods and, in general, weight reduction extremely effectively counteracts NAFLD progression [9].

In details, diet supplements or nutraceutical agents having cellular antioxidant activity are likely to have therapeutic capacities in NAFLD $[9,13]$. For example, in rat fed with high fructose diet, curcumin relieves NAFLD activating Nrf2 signaling [14], while vitamin E significantly reduces the overproduction of ROS induced by fructose [6]. In particular, various clinical trials are underway to demonstrate the effectiveness of vitamin $\mathrm{E}$ in NAFLD management: Vilar-Gomez et al. have demonstrated that vitamin E supplementation improved clinical outcomes in diabetic and no diabetic patients with NASH ameliorating fibrosis or cirrhosis [15]. Moreover, Sanyal et al. have compared vitamin $\mathrm{E}$ and pioglitazone efficacy in liver steatosis observing that vitamin $E$ had a greater effectiveness for the treatment of nonalcoholic steatohepatitis in adults without diabetes [16].

L-Carnitine (LC) plays a critical role in a number of intracellular and metabolic functions, such as fatty acid transport into the mitochondria, stabilization of cell membranes, and reduction of serum lipid levels [17]. Moreover, recent evidence has showed, as LC is also a potent antioxidant: in vitro studies, performing mouse myoblasts [18], rat cardiomyocytes [19] and human osteoblastic cells [20], LC supplementation decrease ROS overproduction and cellular antioxidant defense system.

Based on LC proprieties, Malaguarnera et al. studied how oral LC supplementation improved liver functions and histological patterns in patients with NASH [21]. However, LC mechanism of its protective effect on NAFLD remains to be elucidated and LC action on hepatic damages induced by high fructose intake should be investigated.

The objective of the present study was to clarify the underlying mechanisms of LC action in counteracting fructose-induced hepatic steatosis using human hepatocytes (HepG2 cells). In details, we investigated how LC could regulate fat deposition and mitochondrial anti-oxidative processes.

\section{Materials and methods}

\section{Materials}

All utilized reagents were purchased from Sigma Chemical Co. (St. Louis, MO, U.S.A.).

Primary antibodies against calnexin (H-70), GAPDH (FL335), AMPK $\alpha 1 / 2$ (H-300), pCaMKII (22B1), Nrf2 (C-20), PGC1 $\alpha$ (H-300), SOD2 (FL-222), peroxidase-conjugated secondary antibodies for western blot analysis and rhodamine/FITC-conjugated antibodies for immunofluorescence studies were purchased from Santa Cruz Biotechnology (Santa Cruz-CA, U.S.A.). Primary antibody p-AMPK $\alpha 1 / 2$ (Thr 172) and CaMKII (6G9) was purchased from Cell Signaling Technology (Danvers-MA, U.S.A.).

Compound C (dorsomorphin), AMPK inhibitor, was acquired from Aurogene (Roma, Italy).

\section{Cell line, culture conditions}

The human hepatocellular carcinoma cell line HepG2 was obtained from the European Collection of Cell Cultures (ECACC) and maintained in MEM containing 10\% fetal bovine serum (FBS), $1 \%$ penicillin streptomycin, $1 \%$ glutamine, and 1\% MEM non-essential amino acid. The cells were incubated in a humidified atmosphere of $5 \% \mathrm{CO}_{2}$ at $37{ }^{\circ} \mathrm{C}$ and passaged by trypsinization when they reached $80 \%$ confluence. The culture medium was changed every day, following literature indications. For all experiments, HepG2 were treated with $5 \mathrm{mM}$ fructose $(\mathrm{F})$ and $5 \mathrm{mM}$ L-Carnitine (LC) (single or combined treatment), as indicated in the treatment plan in Fig. 1a. These doses were chosen based on literature data [18, 19]. We studied HepG2 growth capacity to evaluate the absence of cytotoxic effects. Growth curve and cell viability test were performed as previously described. Briefly, HepG2 cells $\left(2 \times 10^{5}\right)$ were plated at $40 \%$ confluence and grown in MEM. The cells were 
B
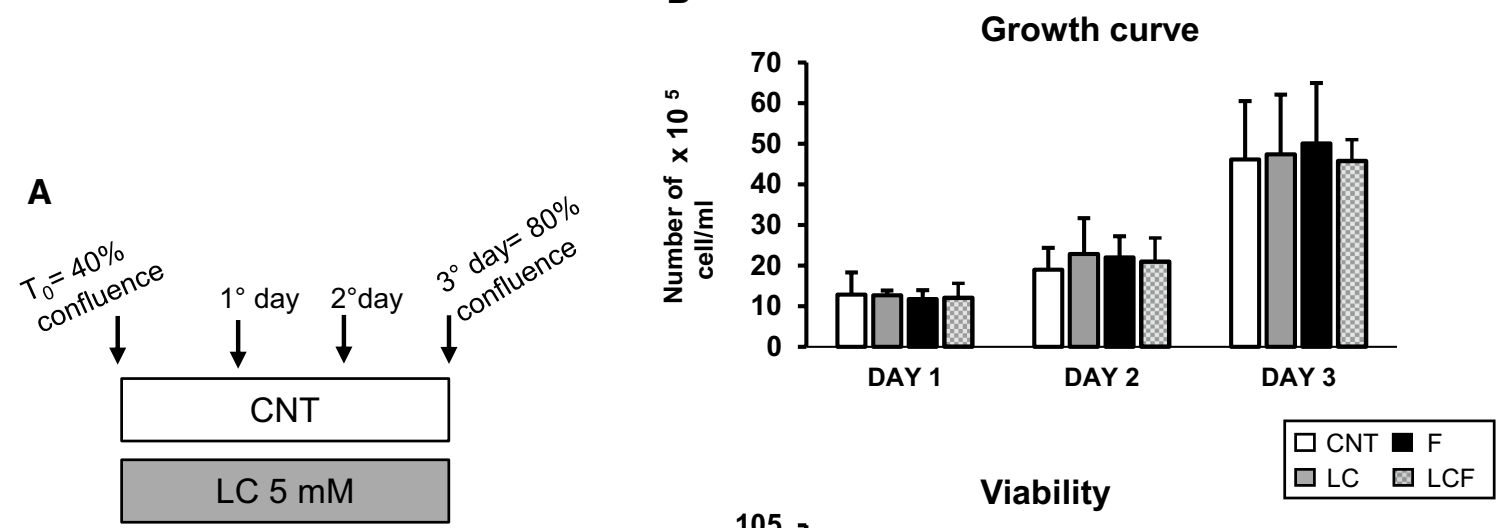

F $5 \mathrm{mM}$
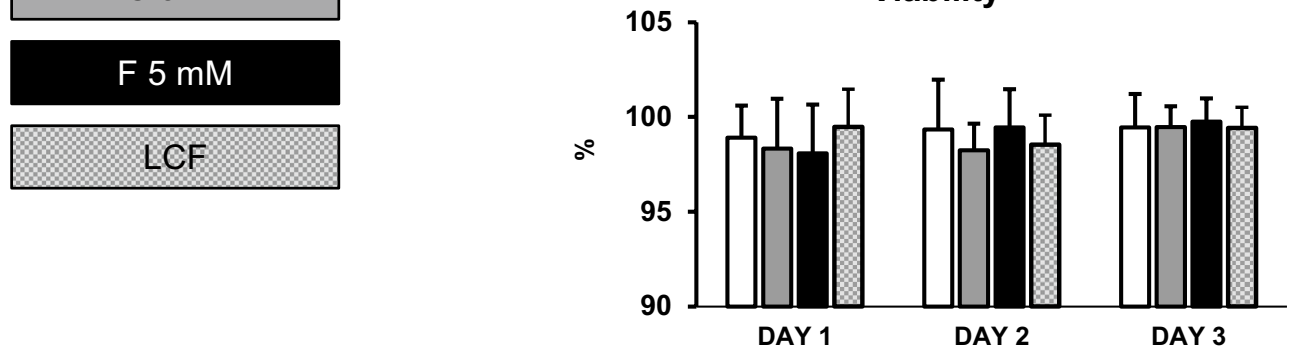

Fig. 1 Treatment action on proliferative phase of HepG2 cells. a Experimental scheme of HepG2 treatments. b Growth curve and viability determination: treatment with LC, F or LCF not altered HepG2 proliferative potential. Viability graph showed the absence of cell

mortality in all treatment conditions. For growth curve and viability, a two-way ANOVA test followed by Tukey's multiple comparison test was performed

treated or not with F and LC for 1, 2 or 3 days. Every day cells were trypsinized, stained with trypan blue, and counted using hemocytometer. Cell viability was calculated by dividing the no stained viable cell count by the total cell count [22]. To abolish AMPK activity, we added AMPK inhibitor, compound $\mathrm{C}$, at $10 \mu \mathrm{M}$ for $72 \mathrm{~h}$ [23].

\section{Western blot analysis}

HepG2 cells were grown in $100 \mathrm{~mm}$ culture dishes with or without LC combined or not with F. Cellular extracts were obtained lysing the cells in RIPA buffer as previously described [24]. Thirty micrograms of proteins were separated by SDS-polyacrylamide gel electrophoreses (SDS-PAGE) and electrophoretically transferred to nitrocellulose membranes (Potran ${ }^{\circledR}$, Whatman ${ }^{\circledR}$ Schleicher \& Schuell). The blots were then blocked and probed with specific primary antibodies, followed by incubation with anti-species-specific secondary antibodies.

To confirm equal protein loading per sample, we used antibody anti-calnexin or anti-GAPDH. Detection of specific proteins was performed by enhanced chemioluminescence reagent (Western Lightning ECL Pro, Perkin Elmer). Quantitative measurement of immunoreactive band intensities was performed by densitometry analysis using the Scion Image software (Scion Corporation, Frederick, MD, USA). Only for AMPK inhibitor experiments and CaMKII, immunoreactive bands were visualized by Uvitec Alliance LD9 gel imaging system (Uvitec, Cambridge, UK).

Data were then presented as ratio between treated cells and control.

\section{Immunofluorescence}

HepG2 cells were grown on coverslips with or without $5 \mathrm{mM}$ LC combined or not with $5 \mathrm{mM} \mathrm{F}$. After $72 \mathrm{~h}$ of treatment, cells were washed with PBS, fixed in $4 \%$ paraformaldehyde and lastly, rinsed three times in PBS. Then, the cells on the coverslips were incubated for $30 \mathrm{~min}$ at room temperature with $1 \%$ bovine serum albumin in PBS with $0.2 \%$ Triton X-100. HepG2 cells on coverslips were then immunostained with specific primary and secondary antibodies, rhodamine- or FITC-conjugated, and nuclei were revealed with DAPI staining.

The MITO CytoPainter mitochondrial indicator (Prodotti Gianni, Italy) is a hydrophobic compound that easily 
A

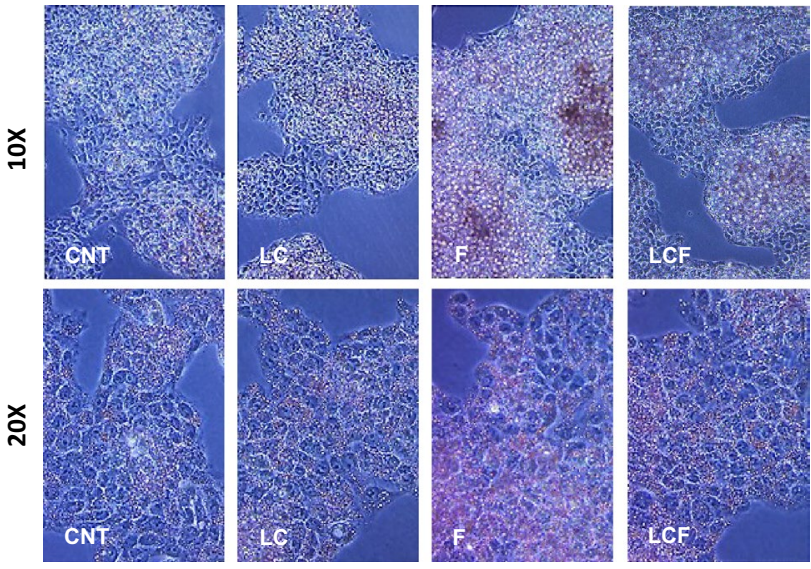

\section{Oil Red O quantification}
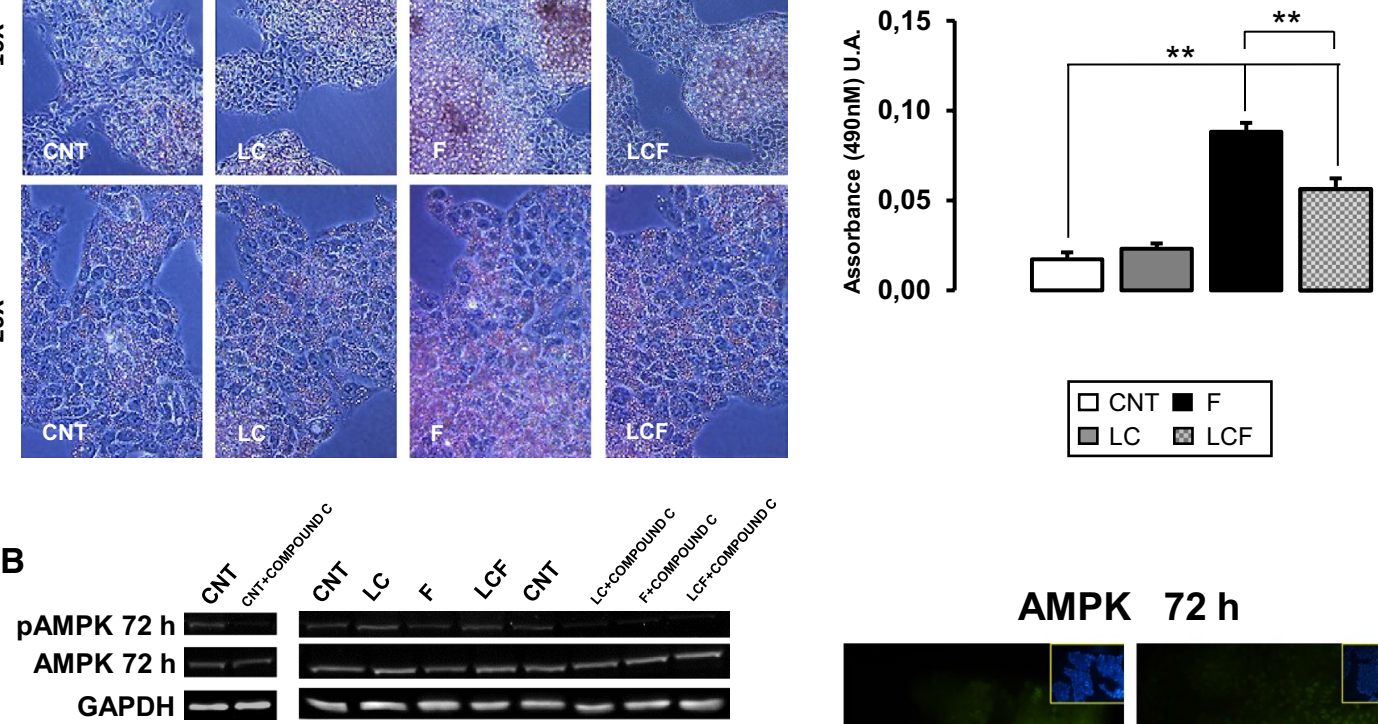

PAMPK/AMPK $72 \mathrm{~h}$
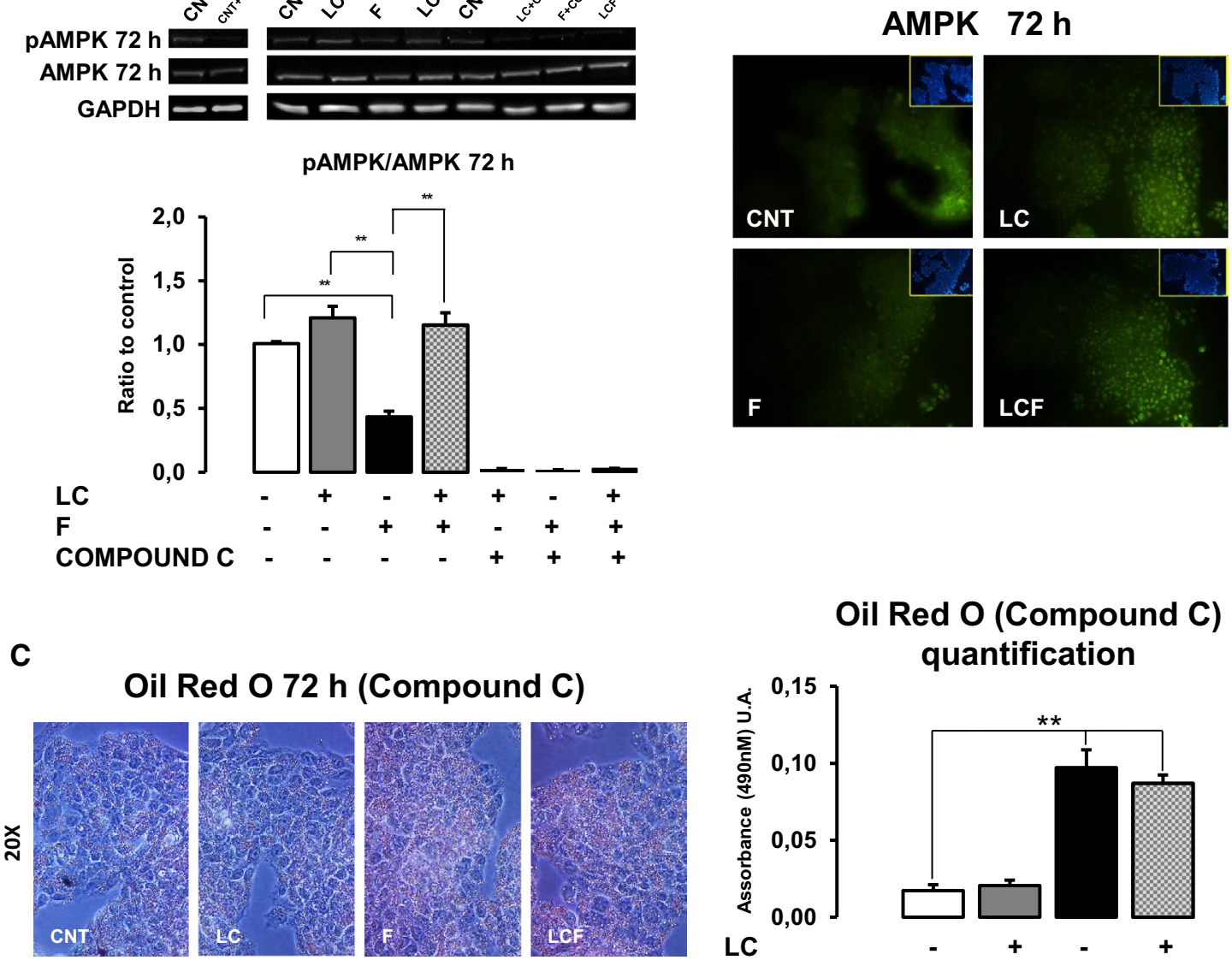

Oil Red O (Compound C)

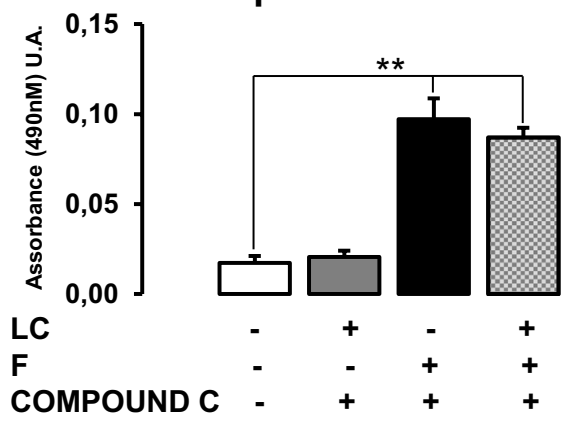

permeates intact live cells remaining trapped in mitochondria. This staining was performed on HepG2 live cells.

Cell ROX ${ }^{\circledR}$ oxidative stress reagents (Thermo Fisher Scientific, Italy) are fluorogenic probes designed to reliably measure reactive oxygen species (ROS) in live cells. The cell-permeable reagents are non-fluorescent or very weak fluorescent, while in a reduced state and upon oxidation exhibit strong fluorogenic signal. Cell ROX ${ }^{\circledR}$ 
4Fig. 2 L-Carnitine role on intracellular lipids deposition. a Oil Red O coloration at $72 \mathrm{~h}$ of treatment showed how LC does not change intracellular fat deposition in respect to CNT. F treated HepG2 showed a higher vesicular lipid deposition compared to CNT, while LC addition diminished lipid accumulation. The stained lipid content was quantified by measuring absorbance at $490 \mathrm{~nm}$. Objective 10X-20X. b Western blot analysis reported LC positive action on AMPK protein activation at $72 \mathrm{~h}$ of treatment: LC counteracted $\mathrm{F}$ action that significantly decreased AMPK activity. LC action was abolished by $10 \mu \mathrm{M}$ Compound $\mathrm{C}$ (an AMPK inhibitor). Immunofluorescence assay showed the increase of AMPK protein expression in HepG2 cells treated with LC alone or in combined condition LCF with respect to $\mathrm{CNT}$ and $\mathrm{F}$ at $72 \mathrm{~h}$ of treatment. Objective: 20X. c Lipid deposition in F and LCF condition, after treatment with $10 \mu \mathrm{M}$ Compound C (an AMPK inhibitor), was superimposable (Fig. 2c). Moreover, in CNT and LC, Compound C did not modify minimal lipid accumulation observed. The stained lipid content was quantified by measuring absorbance at $490 \mathrm{~nm}$. ANOVA-Tukey post test: ${ }^{* *} p \leq 0.01$. Representative immunoblots, obtained using Uvitec Alliance LD9 gel imaging system, are shown

Orange Reagents are localized in the cytoplasm. ROS production by mitochondria was visualized in fluorescence microscopy using the MitoSOX ${ }^{\mathrm{TM}}$ red reagent (Thermo Fisher Scientific, Italy). These staining were performed on HepG2 lived cells after $72 \mathrm{~h}$ of treatment.

Slides were mounted with Moviol. Cells were observed using Nikon Eclipse 50I microscopy and images were captured using Nis-Elements D 4.00 software (Nikon Instruments Europe BV-Netherlands).

\section{Oil Red 0 Staining}

Oil Red O bases this technique on the staining of intracellular lipid droplets.

After treatments with LC and F, HepG2 cells were washed with PBS and fixed using $4 \%$ formalin for $30 \mathrm{~min}$. Subsequently, the cells were washed twice-using $\mathrm{ddH}_{2} \mathrm{O}$ and incubated with Oil Red O solution for $15 \mathrm{~min}$ at RT. To remove background staining, the cells were washed three times with a $60 \%$ isopropanol solution for $5 \mathrm{~min}$. For quantitative analysis of Oil Red O contents levels, isopropanol was added to each samples and then shaken at room temperature for $5 \mathrm{~min}$. The absorbance was read at $490 \mathrm{~nm}$ with Bio-rad iMark Microplate Reader (Bio-Rad Laboratories, Inc., Hercules, CA, USA).

\section{Statistical analysis}

All experiments were performed three times. All data are presented as mean $\pm \mathrm{SD}$.

Shapiro-Wilk test was used to ascertain normality of the data distribution.

For variables with Gaussian distribution, one-way analysis of variance (ANOVA) was used to compare means among the groups (followed by Tukey's post hoc test), whereas for variables with non-Gaussian distribution, Kruskal-Wallis test was used to compare means among the groups (followed by Dunn's post hoc test). Results were considered significant when $p \leq 0.05$.

Statistical analysis was performed with specific statistical packages (Prism v 7.00 GraphPad Software, San Diego, CA, USA).

\section{Results}

In this study, the model of steatosis was mimicked using human hepatocytes HepG2 treated with $5 \mathrm{mM} \mathrm{F}$ for $72 \mathrm{~h}$ (Fig. 1a). We first verified that $5 \mathrm{mM} \mathrm{F}, 5 \mathrm{mM} \mathrm{LC}$ and combination of $\mathrm{F}$ and LC did not cause cytotoxic effects. As shown in Fig. 1b, these treatments did not modify growth curve and HepG2 vitality. We, therefore, investigated F, LC and LCF action on lipid accumulation.

\section{HepG2 intracellular lipid deposition}

After $72 \mathrm{~h}$, in CNT condition, a minimal lipid deposition occurred, as expected [5], and HepG2 cells treated with LC displayed a level of lipid superimposable to CNT (Fig. 2a). F-treated HepG2 showed a higher vesicular lipid deposition compared to CNT, while LC addition blunted lipid accumulation, as revealed by Oil Red quantification (Fig. 2a).

\section{L-Carnitine effects on AMPK}

We hypothesized that lipid droplets increase was linked to AMPK activity.

Figure $2 \mathrm{~b}$ shows that $\mathrm{F}$ significantly decreased AMPK activity, while LC supplementation counteracted this alteration. Above all, when HepG2 were treated with $10 \mu \mathrm{M}$ compound $\mathrm{C}$ (an AMPK inhibitor), lipid deposition in F and LCF condition was superimposable (Fig. 2c). Moreover, in CNT and LC, compound $\mathrm{C}$ did not modify minimal lipid accumulation observed. At the same time, we observed that AMPK activity is abolished in F, LC and LCF conditions (Fig. 2b).

\section{L-Carnitine effects on CaMKII}

AMPK can be activated by intracellular calcium levels, in the same manner of calcium/calmodulin-dependent protein kinase II (CaMKII) [25] and our group has recently demonstrated that LC could regulate the intracellular calcium levels [26]. As shown in Fig. 3, $72 \mathrm{~h}$ of LC treatment stimulates CaMKII protein expression and increases CaMKII activation. In F-treated HepG2 cells, CaMKII activation is not 


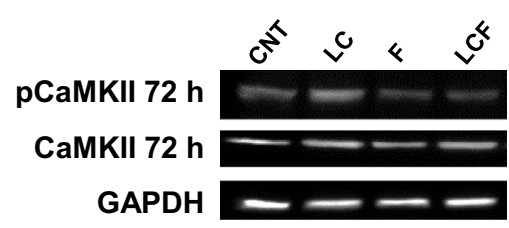

pCaMKII/CaMKII $72 \mathrm{~h}$
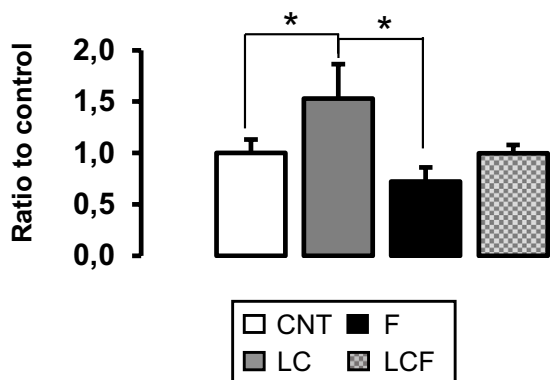

CaMKII $72 \mathrm{~h}$
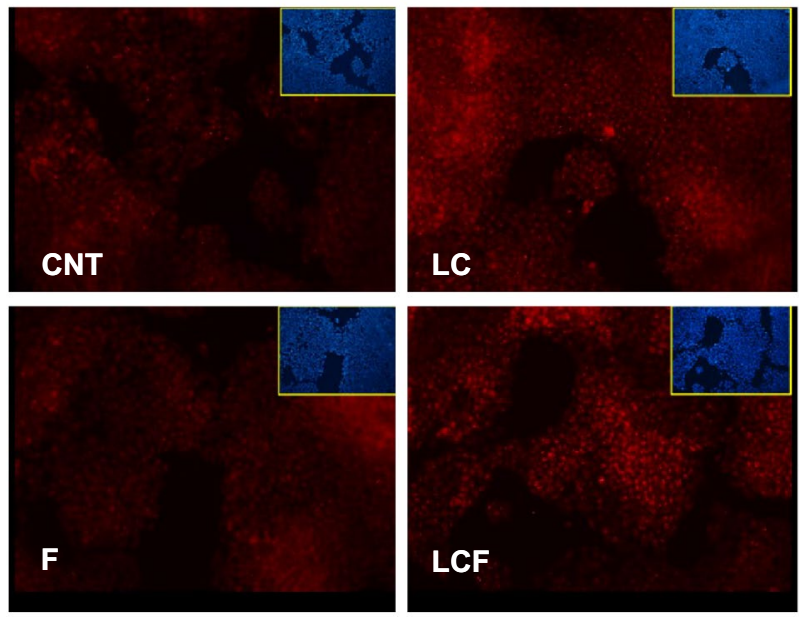

Fig. 3 L-Carnitine role on CaMKII protein content. Immunofluorescence and western blot assay at $72 \mathrm{~h}$ of treatment showed how LC promoted CaMKII protein expression in $\mathrm{HepG} 2$ in respect to CNT

modified compared to CNT and the same effect is observed in LCF condition.

\section{L-Carnitine effects on mitochondrial biogenesis and anti-oxidative response}

Our group has recently demonstrated that LC could regulate cellular anti-oxidative responses [18-20, 26]. Since mitochondria are the primary intracellular regulator of ROS balance, LC effect on mitochondrial activity in HepG2 cells was studied. Figure $4 \mathrm{a}$ revealed that after $72 \mathrm{~h}$ of treatment, $\mathrm{LC}$ seems to be able to increase fluorescence signal linked to mitochondria in respect to CNT, while F seems decreases it. Peroxisome proliferator-activated receptor gamma coactivator $1(\mathrm{PGC} 1 \alpha)$ is a key regulator of mitochondrial dynamics and biogenesis [27]. Figure $4 \mathrm{~b}$ shows how $72 \mathrm{~h}$ of LC treatment significantly increased PGC1 protein levels in respect to $\mathrm{CNT}$ and F: LC counteracted PGC1 $\alpha$ expression inhibition by $\mathrm{F}$ in LCF condition. Moreover, when HepG2 were treated with Compound $\mathrm{C}$ for $72 \mathrm{~h}, \mathrm{PGC} 1 \alpha$ protein increase is completely inhibited in all conditions (Fig. 4c), showing an AMPK-dependent LC and F activity on PGC1.

These results suggested that $\mathrm{LC}$, both alone and in the presence of fructose, could improve mitochondria ROS production. To verify this speculation, we evaluated, with a specific fluorescence probe (MitoSOX ${ }^{\mathrm{TM}}$ ), mitochondrial ROS production. As reported in Fig. 4d, in F-treated HepG2 fluorescence intensity is increased in respect to CNT and LC, while in LCF condition, ROS signal is attenuated. In HepG2 treated with LC, ROS label is similar to CNT. and in respect to $\mathrm{F}$ in combined condition (LCF). Objective: 20X. Representative immunoblots, obtained using Uvitec Alliance LD9 gel imaging system, are shown

Additionally, we investigated whether LC antioxidant effect is limited to mitochondria or involved other cellular compartments. Using ROX Cell ROX ${ }^{\circledR}$ Oxidative Stress Reagents, we observed that after 30 min of $\mathrm{H}_{2} \mathrm{O}_{2}$ stress, ROS production was reduced following $72 \mathrm{~h}$ of pre-treatment with LC, as shown in Fig. 5a. In details, LC seems to be able to decrease ROS response both alone (LC) and in the presence of fructose (LCF).

Finally, LC supplementation significantly increased SOD2 protein levels after $48 \mathrm{~h}$ and $72 \mathrm{~h}$ of treatment, as reported in western blot assay (Fig. 5b). Furthermore, in F condition, SOD2 protein levels were significant decreased, while LC counterbalanced F effects in HepG2 cells treated with combined stimuli (LCF).

Moreover, in Fig. 5b, western blot assay showed how LC treatment significantly increased Nrf2 protein content in respect to CNT and F stimulation.

Furthermore, Fig. $5 \mathrm{c}$ shows that $72 \mathrm{~h}$ compound $\mathrm{C}$ treatment significantly decreased LC positive action on SOD2 and $\mathrm{Nrf} 2$, indicating an AMPK-dependent modulation by LC. In details, SOD2 showed a minimal residual expression in all three conditions (LC, F, LC +F), suggesting both an AMPK-dependent and independent modulation. While, NRF2 protein increase was significantly inhibited by AMPK inhibitor in LC-treated HepG2, but it maintained a residual expression in $\mathrm{F}$ presence, suggesting that $\mathrm{F}$ could also enhance NRF2 content in an AMPK-independent manner. 
A MITO ASSAY $(72 \mathrm{~h})$
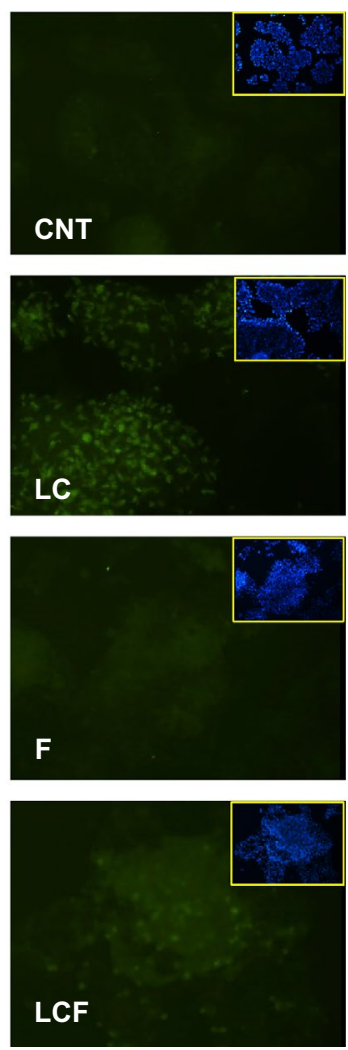

C

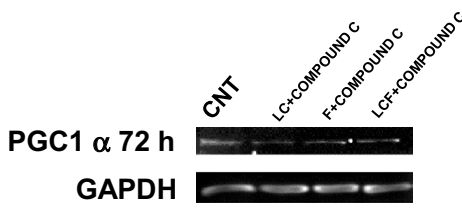

B

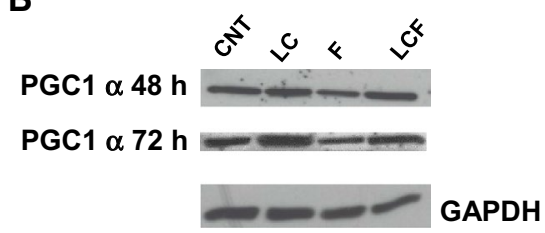

PGC1 $\propto 48 \mathrm{~h}$
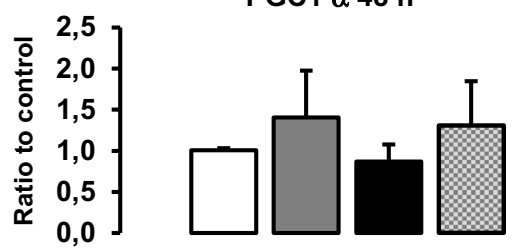

PGC1 $\propto 72 \mathrm{~h}$
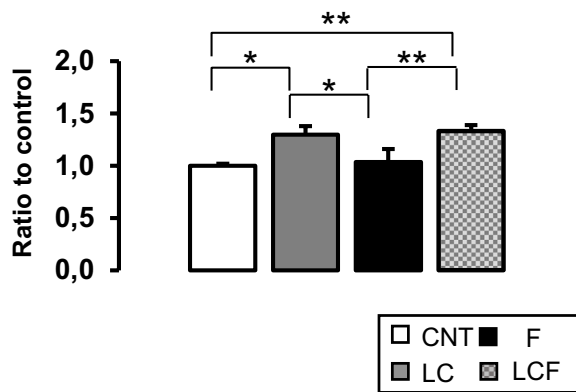

MitoSOX ASSAY (72 h)
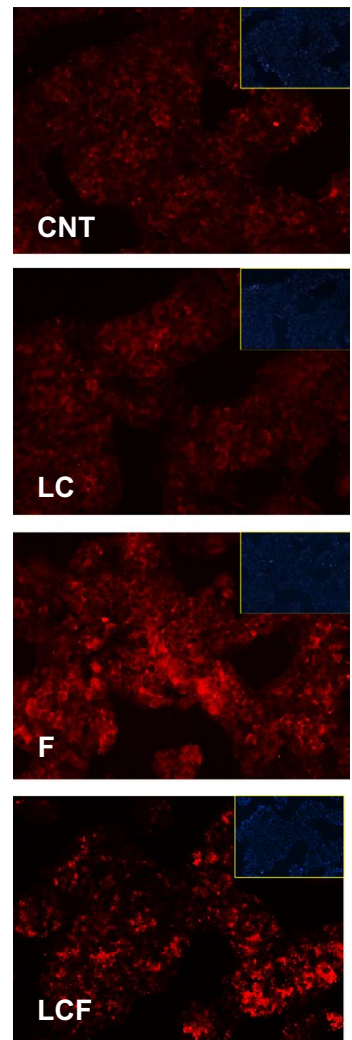

PGC1 $\propto 72$ h-Compound C
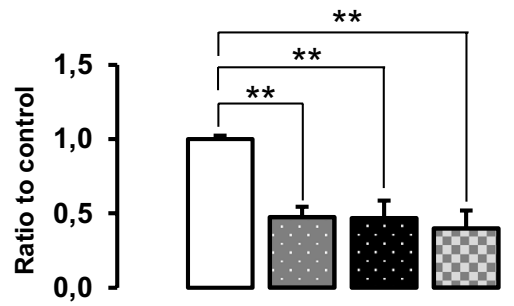

Fig. 4 L-Carnitine role on mithocondria. a MITO CytoPainter staining revealed that, at $72 \mathrm{~h}$ of treatment, $\mathrm{LC}$ seems to be able to rise the signal of activated mitochondria in respect to CNT and in respect to combined condition (LCF). Objective 20X. b Western blot quantification reported PGC1 $\alpha$ increase after LC treatment. This action resulted significant after $72 \mathrm{~h}$ of treatment. It is important to note how this effect resulted also corroborated in the combined condition (LCF), in which LC can counteract F action. $\mathbf{c}$ Western blot analy-

\section{Discussion}

Excessive consumption of fructose, especially sugar-sweetened beverages, is strictly associated with the development of liver steatosis $[4,5]$. NAFLD is emerging as a primary health problem in the world also considering its association with diabetes and cardiovascular disease $[1,3]$. sis of PGC1 $\alpha$ after $72 \mathrm{~h}$ of treatments performed in association with AMPK inhibitor: LC positive action on PGC1 $\alpha$ protein content was abolish indicated that LC action is AMPK-mediated. d Mito SOX staining shown that, at $72 \mathrm{~h}$ of treatment, LC does not increase the level of mitochondrial ROS compared to CNT. In combination with F, LC appears to be able to reduce the levels of mitochondrial ROS compared to F. ANOVA-Tukey post test: $* p \leq 0.05$, * $* \leq 0.01$. Representative immunoblots are shown

Now, specifically therapies for NAFLD are limited [10-12] and currently lifestyle changes, i.e. diet modifications and physical activity enlargement, are effective in the treatment of liver steatosis [9]. However, several patients are not agreeable with healthy these indications. Consequently, finding drugs or natural compound that are more useful for NAFLD therapy has become one of the hottest research fields [13]. 
A

\section{ROS production $(72 \mathrm{~h})$}

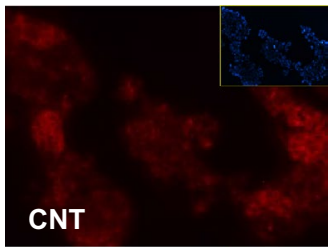

B
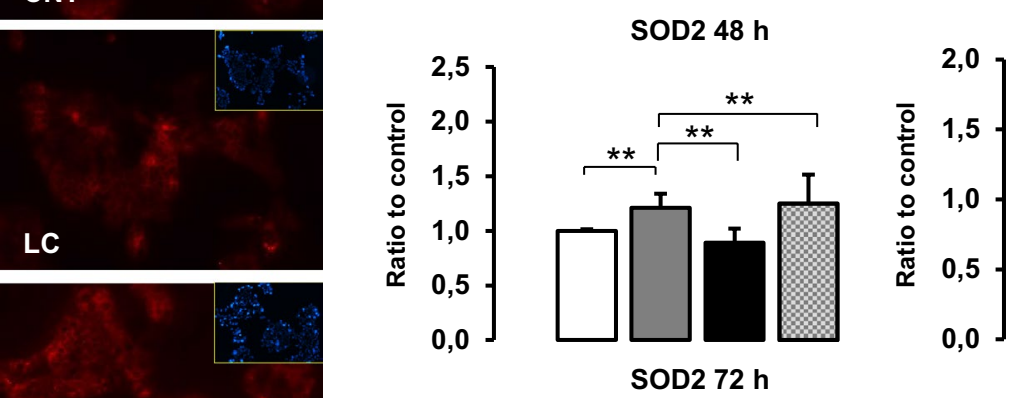

Nrf2 48 h
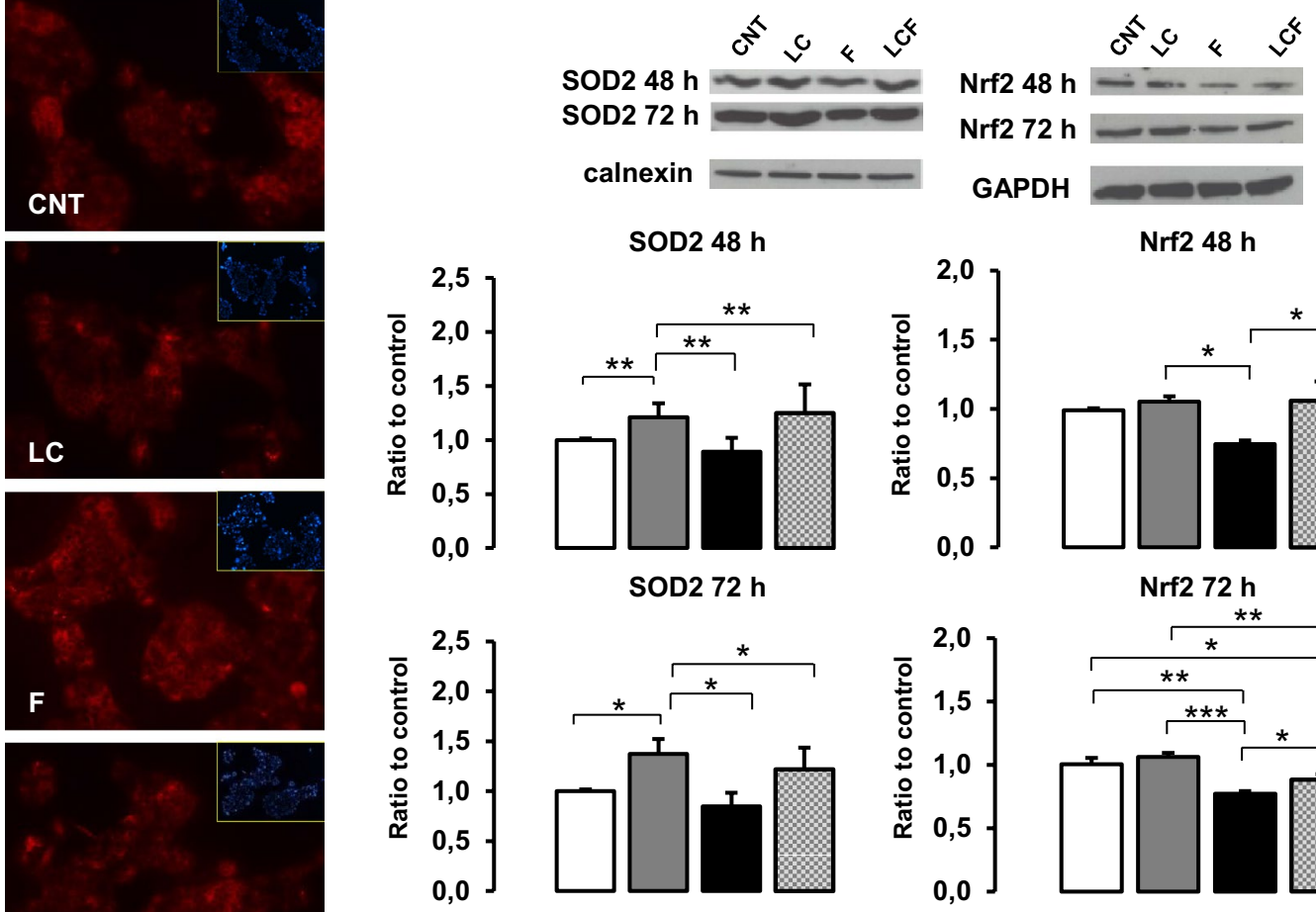

GAPDH
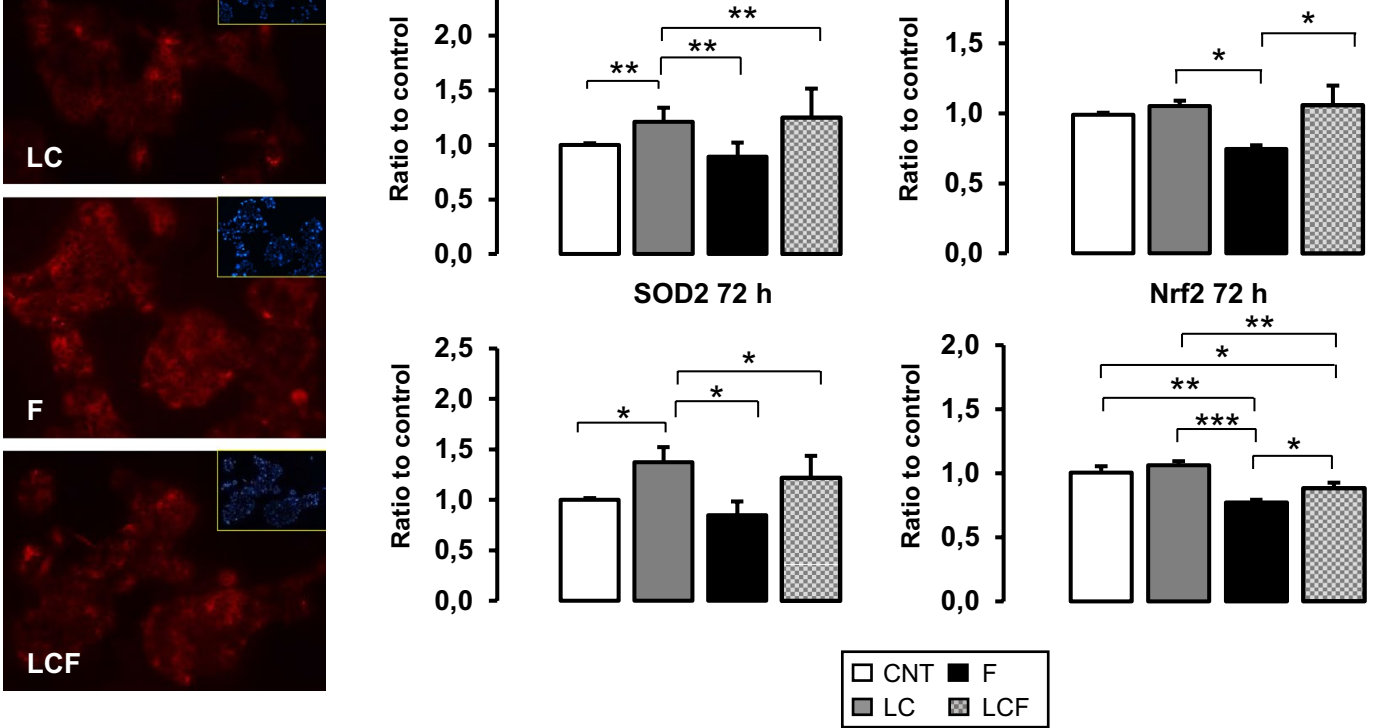

C
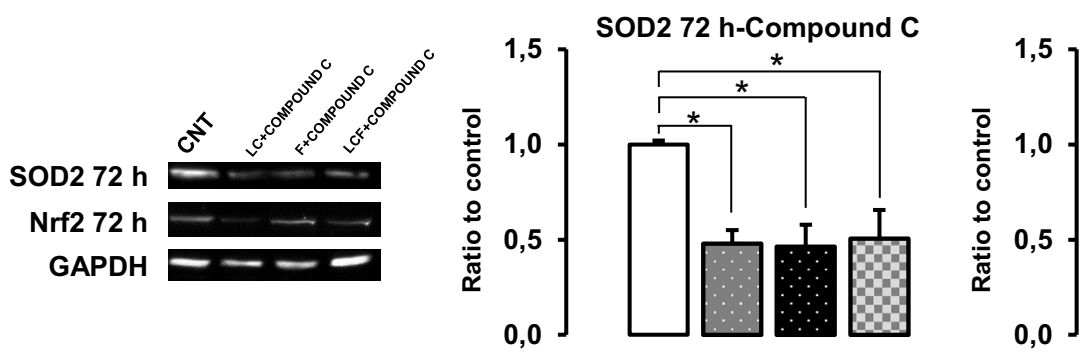

Nrf2 72 h-Compound C

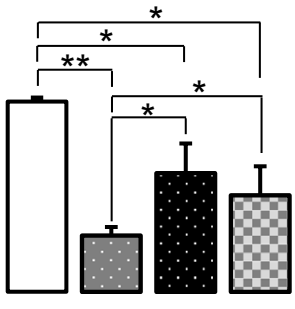

\begin{tabular}{|lc|}
\hline$\square$ CNT & F+COMPOUND C \\
$\square$ LC+COMPOUND C $\square$ LCF+COMPOUND C
\end{tabular}

Fig. 5 L-Carnitine role on ROS production and antioxidant factors. a Cell ROX assay after 30 min of $\mathrm{H} 2 \mathrm{O} 2$ stress stimulus showed LC protective action in respect to CNT: $\mathrm{LC}$ treatment decreased reactive oxygen species production in HepG2 cells after $72 \mathrm{~h}$ of treatment. This action was confirmed also in the combined condition (LCF). Objective: 20X. b Western blot analysis of SOD2 and Nrf2 protein content after 48 and $72 \mathrm{~h}$ of treatment. LC significant promoted SOD2 protein levels in HepG2 in respect to control and in respect to $\mathrm{F}$ in the combined condition (LCF). In the similar manner, LC increased $\mathrm{Nrf} 2$ protein content after $48 \mathrm{~h}$ and $72 \mathrm{~h}$ of treatment alone and in combined condition (LCF). $\mathbf{c}$ Western blot analysis of SOD2 and Nrf2 protein content after $72 \mathrm{~h}$ of treatments $\mathrm{LC}, \mathrm{F}$ and $\mathrm{LC}+\mathrm{F}$ in association with AMPK inhibitor Compound C: SOD2 showed a minimal residual expression in all three conditions suggesting that its expression is regulated in AMPK-dependent and independent manner. Nrf2 protein content is significantly decreased in LC treated cells, while a residual expression was present in $\mathrm{F}$ condition suggesting that $F$ could also promotes Nrf2 content in an AMPK-independent manner. ANOVA-Tukey post test: $* p \leq 0.05, * * p \leq 0.01$, $* * * p \leq 0.001$. Representative immunoblots are shown 
We investigated in fructose-induced steatosis HepG2 cells, the effects of $5 \mathrm{mM} \mathrm{LC}$ to clarify LC action in countering NAFLD.

Our data demonstrate that LC supplementation can reduce HepG2 intracellular fructose-induced lipid accumulation, recovering AMPK activity suppressed by fructose treatment (Fig. 2a). Use of an AMPK inhibitor (compound C) abolishes LC effect, confirming AMPK-mediated LC action (Fig. 2c). Recently, Woods et al. have revealed, using transgenic mice with AMPK tissue conditional expression, that liver-specific activation of this kinase reduces lipogenesis in vivo and entirely protects against liver steatosis induced by high-fructose diet [5]. Moreover, several studies have shown how different nutraceuticals, i.e. pterostilbene or paeoniflorin, could reduce lipid accumulation in rats/mice fed high fructose diet by activating AMPK signaling and thus, promoting $\beta$-oxidation and inhibiting lipogenesis [28, 29].

Furthermore, based on the closed correlation between excessive fructose intake, insulin resistance, and NAFLD, several studies indicated that metformin, an AMPK activator, might have therapeutic potential on liver steatosis [30]. Moreover, data obtained by increasing number of works suggest how SGLT2 inhibitors are an attractive option for NAFLD pharmacologic management [31]. Interestingly, Mancini et al. have demonstrated that canagliflozin, a SGL2 inhibitor used in patients with type 2 diabetes, activates AMPK in different cell lines and in mouse liver [32, 33]. Therefore, LC, used in associated with anti-diabetes drugs, could represent a beneficial therapeutic option for NAFLD.

Additionally, AMPK is a $\mathrm{Ca}^{2+}$-related protein: its activation may be due to calcium oscillations [34]. We speculated that LC could play an important role on calcium signaling and, to verify our hypothesis, we analyzed CaMKII protein level detecting that LC promoted CaMKII expression and activation (Fig. 3). These results reveal that LC may participate in the regulation of calcium signaling pathways confirming our previous data that revealed how LC influences calcium release from intracellular calcium stores in human osteoblasts [26]. Accumulating evidence of the role of this nutraceutical on calcium homeostasis opens up new therapeutic developments in the pathologies characterized by a calcium impairment, including diabetes mellitus and cardiovascular diseases.

Numerous data suggest that chronic exposure to a highfructose microenvironment could lead to a decrease in the mitochondrial biogenesis and a concomitant increase in mitochondrial oxidative stress production [35] in hepatocytes.

Our data indicated that LC treatment could counteract fructose action on mitochondrial biogenesis enhancing PGC1 $\alpha$ protein content in steatosis HepG2 (Fig. 4b). As known, PGC1 $\alpha$, the master regulator of mitochondrial biogenesis, is primary regulated by AMPK [36]. Therefore, LC could maintain mitochondrial homeostasis through AMPK activation and subsequently, PGC1 $\alpha$ synthesis as indicated by results obtained using AMPK inhibitor (compound C) (Fig. 4b, c). This action could attenuate environmental stress caused by fructose.

As before mentioned, oxidative stress is critically involved in NAFLD pathogenesis, because caused lipid peroxidation increasing ROS production. In particular, high fructose micro-environmental stimulates ROS formation $[4,6,7,35]$ and this study confirmed the exacerbated ROS production not only in mitochondria, but also in other cytoplasm compartments (Fig. 4c). Our data reveled as LC had an antioxidant effect on steatosis HepG2 cells: ROS production was alleviated in mitochondria and in cytoplasm. Moreover, the protein content of SOD2 and Nrf2, the main factors involved in cellular antioxidant responses, were increased in LCF condition in respect to F (Fig. 5b).

SOD2, an important antioxidant enzyme present in hepatocytes, thwarts oxidative damages. In fact, it was shown that SOD knockout mice presented abnormal lipid metabolism in the liver. High levels of oxidative stress, caused by defects in the antioxidant system as result of concomitant deficiency of SOD in SOD-knockout mice, resulted in hepatic lipid accumulation via altered lipid metabolism [37].

Our data, obtained by western blot analysis (Fig. 5b, c) showed an incremental LC effect on SOD2, which is inhibited by compound $\mathrm{C}$, although the permanence of a very weak signal suggests a $L C$ residual activity through an independent AMPK mechanism. LC increased SOD2 protein concentration compared to both $\mathrm{CNT}$ and $\mathrm{F}$, but its effect is not enough to counteract $\mathrm{F}$ presence, supporting the hypothesis that other AMPK-independent pathways are at stake. This evidences agrees with the dichotomous SOD2 regulation described in carcinogenic cells, like HepG2 cell line [38] and support our date showing that $F$ seems to act on SOD2 through both AMPK-dependent and -independent manner.

Various studied have shown that fructose downregulated Nrf2 expression and that some natural products ameliorate liver steatosis by reverting this effect [14, 37, 39]. In agreement with these observations, in our experiment, fructose decreased Nrf2 content while LC treatment recovered Nrf2 protein level (Fig. 5b). More importantly, many works have revealed the crucial role of Nrf2 in progression of NAFLD to steatohepatitis: in mice, Nrf2 depletion increased ROS production and consequently, liver fibrosis and inflammation, the principal pathological features of NASH [37].

It is also important to highlight that numerous data have indicated how Nrf2 expression is regulated by AMPK activation [40] and showed by our results obtained using AMPK 
inhibitor (Fig. 5c). Consequently, our results suggested that LC-promoting AMPK activity enhances a synergic action on mitochondrial homeostasis and oxidative defense machine that could counteract fructose-induced hepatic steatosis.

Interestingly, the results of this work point out on LC antioxidant properties. In line with an increasing number of evidence, our results showed LC ability to reduce ROS production and contemporarily to rise the expression of antioxidant enzymes, i.e. SOD, catalase and phospholipid hydroperoxide glutathione peroxidase in an in vitro, in vivo models and humans [41-44]. In this regard, our group established this LC ability in skeletal muscle cells model, cardiomyocytes and osteoblastic cells observing that this anti-oxidant action is strictly correlated with calcium signaling [18-20, 26, 41]. Additional investigations are needed to better clarify LC antioxidant function and mechanisms.

\section{Conclusion}

This experimental study on human hepatocytes was designed to verify the protective role of LC against fructose-induced liver steatosis and to investigate LC molecular mechanisms of action. In this regard, we studied lipid deposition, mitochondrial homeostasis and antioxidant status, thus determining AMPK activity and CaMKII, SOD2 and Nrf2 level.

Taken together, our findings indicated that LC succeeds, through AMPK activation, in attenuating fructose-induced lipid deposition and, by regulating SOD and Nrf2 activity, preserves mitochondrial homeostasis and strengths cellular antioxidant machine in human HepG2 cells.

This evidence highlights the potential protective effect of this nutraceutical compound in NAFLD.

\begin{abstract}
Authors' contributions AM, PS, and FV contributed equally to this work. AM, PS, and FV designed, performed experiments, analyzed data and wrote the manuscript. LL, SB, MM, and GM contributed to the discussion and reviewed the manuscript. IT supervised, designed experiments and wrote the manuscript. IT is the guarantor of this work and takes responsibility for the integrity of data and the accuracy of data analysis. All authors read and approved the final manuscript.
\end{abstract}

Funding This study was partially supported by Ricerca Corrente funding from Italian Ministry of Health to IRCCS Policlinico San Donato.

\section{Compliance with ethical standards}

Conflict of interest The authors declare that they have no competing interests.

Ethical approval This article does not contain any studies with human participants or animals performed by any of the authors.
Informed consent Informed consent is not required in this type of study.

Open Access This article is distributed under the terms of the Creative Commons Attribution 4.0 International License (http://creativeco mmons.org/licenses/by/4.0/), which permits unrestricted use, distribution, and reproduction in any medium, provided you give appropriate credit to the original author(s) and the source, provide a link to the Creative Commons license, and indicate if changes were made.

\section{Bibliography}

1. Byrne CD, Targher G (2015) NAFLD: a multisystem disease. J Hepatol 62(1 Suppl):S47-S64. https://doi.org/10.1016/j. jhep.2014.12.01

2. Bessone F, Razori MV, Roma MG (2018) Molecular pathways of nonalcoholic fatty liver disease development and progression. Cell Mol Life Sci. https://doi.org/10.1007/s00018-018-2947-0

3. Polyzos SA, Kountouras J, Mantzoros CS (2018) Obesity and nonalcoholic fatty liver disease: from pathophysiology to therapeutics. Metabolism S0026-0495(18):30253. https://doi.org/10.1016/j. metabol.2018.11.014

4. Jensen T, Abdelmalek MF, Sullivan S et al (2018) Fructose and sugar: a major mediator of non-alcoholic fatty liver disease. J Hepatol 68(5):1063-1075. https://doi.org/10.1016/j. jhep.2018.01.019

5. Woods A, Williams JR, Muckett PJ et al (2017) Liver-specific activation of AMPK prevents steatosis on a high-fructose diet. Cell Rep 18(13):3043-3051. https://doi.org/10.1016/j.celrep.2017.03.011

6. Zhang X, Zhang JH, Chen XY et al (2015) Reactive oxygen species-induced TXNIP drives fructose-mediated hepatic inflammation and lipid accumulation through NLRP3 inflammasome activation. Antioxid Redox Signal 22(10):848-870. https://doi. org/10.1089/ars.2014.5868

7. Nigro D, Menotti F, Cento AS et al (2017) Chronic administration of saturated fats and fructose differently affect SREBP activity resulting in different modulation of Nrf2 and Nlrp3 inflammasome pathways in mice liver. J Nutr Biochem 42:160171. https://doi.org/10.1016/j.jnutbio.2017.01.010

8. Sharma RS, Harrison DJ, Kisielewski D et al (2017) Experimental nonalcoholic steatohepatitis and liver fibrosis are ameliorated by pharmacologic activation of Nrf2 (NF-E2 p45-related factor 2). Cell Mol Gastroenterol Hepatol 5(3):367-398. https ://doi.org/10.1016/j.jcmgh.2017.11.016

9. Romero-Gómez M, Zelber-Sagi S, Trenell M (2017) Treatment of NAFLD with diet, physical activity and exercise. J Hepatol 67(4):829-846. https://doi.org/10.1016/j.jhep.2017.05.016

10. Geddawy A, Hussian M, Kamel MY, Kamal R, Ibrahim MA (2017) Effects of liraglutide and vitamin E in fructose-induced metabolic syndrome in rats. Pharmacology 99(1-2):48-56. https ://doi.org/10.1159/000449429

11. Carr RM, Reid AE (2015) FXR agonists as therapeutic agents for non-alcoholic fatty liver disease. Curr Atheroscler Rep 17(4):500. https://doi.org/10.1007/s11883-015-0500-2

12. Hu YB, Liu XY, Zhan W (2018) Farnesoid X receptor agonist INT-767 attenuates liver steatosis and inflammation in rat model of nonalcoholic steatohepatitis. Drug Des Dev Ther 12:22132221. https://doi.org/10.2147/DDDT.S170518

13. Chen Q, Wang T, Li J, Wang S, Qiu F, Yu H, Zhang Y, Wang T (2017) Effects of natural products on fructose-induced nonalcoholic fatty liver disease (NAFLD). Nutrients 9(2):E96. https ://doi.org/10.3390/nu9020096 
14. Yan C, Zhang Y, Zhang X, Aa J, Wang G, Xie Y (2018) Curcumin regulates endogenous and exogenous metabolism via Nrf2-FXR-LXR pathway in NAFLD mice. Biomed Pharmacother 105:274-281. https://doi.org/10.1016/j.biopha.2018.05.135

15. Vilar-Gomez E, Vuppalanchi R, Gawrieh S, Ghabril M, Saxena R, Cummings OW, Chalasani N (2018) Vitamin E improves transplant-free survival and hepatic decompensation among patients with nonalcoholic steatohepatitis and advanced fibrosis. Hepatology. https://doi.org/10.1002/hep.30368

16. Sanyal AJ, Chalasani N, Kowdley KV, McCullough A, Diehl AM, Bass NM, Neuschwander-Tetri BA, Lavine JE, Tonascia J, Unalp A, Van Natta M, Clark J, Brunt EM, Kleiner DE, Hoofnagle JH, Robuck PR, NASH CRN (2010) Pioglitazone, vitamin E, or placebo for nonalcoholic steatohepatitis. N Engl J Med 362(18):1675-1685. https://doi.org/10.1056/nejmoa0907929

17. Adeva-Andany MM, Calvo-Castro I, Fernández-Fernández $C$ et al (2017) Significance of L-carnitine for human health. IUBMB Life 69(8):578-594. https://doi.org/10.1002/iub.1646

18. Montesano A, Senesi P, Luzi L, Benedini S, Terruzzi I (2015) Potential therapeutic role of L-carnitine in skeletal muscle oxidative stress and atrophy conditions. Oxid Med Cell Longev 2015:646171. https://doi.org/10.1155/2015/646171

19. Vacante F, Senesi P, Montesano A, Frigerio A, Luzi L, Terruzzi I (2018) L-carnitine: an antioxidant remedy for the survival of cardiomyocytes under hyperglycemic condition. J Diabetes Res. https://doi.org/10.1155/2018/4028297

20. Terruzzi I, Montesano A, Senesi P et al (2019) L-carnitine reduces oxidative stress, and promotes cells differentiation and bone matrix proteins expression in human osteoblastlike cells. Biomed Res Int 2019:5678548. https://doi. org/10.1155/2019/5678548

21. Malaguarnera M, Gargante MP, Russo C et al (2010) L-carnitine supplementation to diet: a new tool in treatment of nonalcoholic steatohepatitis-a randomized and controlled clinical trial. Am J Gastroenterol 105(6):1338-1345. https://doi.org/10.1038/ ajg.2009.719

22. Terruzzi I, Montesano A, Senesi P, Vacante F, Benedini S, Luzi L (2017) Erratum to: ranolazine promotes muscle differentiation and reduces oxidative stress in $\mathrm{C} 2 \mathrm{C} 12$ skeletal muscle cells. Endocrine 58(1):46. https://doi.org/10.1007/s12020-017-1243-3

23. Wang C, Li Y, Hao M et al (2018) Astragaloside IV inhibits triglyceride accumulation in insulin-resistant HepG2 cells via AMPK-induced SREBP-1c phosphorylation. Front Pharmacol 9:345. https://doi.org/10.3389/fphar.2018.00345

24. Vacante F, Senesi P, Montesano A et al (2019) Metformin counteracts HCC progression and metastasis enhancing KLF6/p21 expression and downregulating the IGF axis. Int J Endocrinol 2019:7570146. https://doi.org/10.1155/2019/7570146

25. Seo WD, Lee JH, Jia Y et al (2015) Saponarin activates AMPK in a calcium-dependent manner and suppresses gluconeogenesis and increases glucose uptake via phosphorylation of CRTC2 and HDAC5. Bioorg Med Chem Lett 25(22):5237-5242. https://doi. org/10.1016/j.bmcl.2015.09.057

26. Ferraretto A, Bottani M, Villa I et al (2018) L-carnitine activates calcium signaling in human osteoblasts. J Funct Foods 47:270278. https://doi.org/10.1016/j.jff.2018.05.068

27. García-Ruiz C, Fernández-Checa JC (2018) Mitochondrial oxidative stress and antioxidants balance in fatty liver disease. Hepatol Commun 2(12):1425-1439. https://doi.org/10.1002/hep4.1271

28. Kosuru R, Kandula V, Rai U, Prakash S, Xia Z, Singh S (2018) Pterostilbene decreases cardiac oxidative stress and inflammation via activation of AMPK/Nrf2/HO-1 pathway in fructose-fed diabetic rats. Cardiovasc Drugs Ther 32(2):147-163. https://doi. org/10.1007/s10557-018-6780-3

29. Li YC, Qiao JY, Wang BY, Bai M, Shen JD, Cheng YX (2018) Paeoniflorin ameliorates fructose-induced insulin resistance and hepatic steatosis by activating LKB1/AMPK and AKT pathways. Nutrients 10(8):E1024. https://doi.org/10.3390/nu10081024

30. Green CJ, Marjot T, Tomlinson JW, Hodson L (2018) Of mice and men: is there a future for metformin in the treatment of hepatic steatosis? Diabetes Obes Metab. https://doi.org/10.1111/dom.13592

31. Akuta N, Kawamura Y, Watanabe C et al (2018) Impact of SGLT2 inhibitor to histological features and glucose metabolism of nonalcoholic fatty liver disease complicated by diabetes mellitus. Hepatol Res. https://doi.org/10.1111/hepr.13304

32. Mancini SJ, Boyd D, Katwan OJ et al (2018) Canagliflozin inhibits interleukin-1 $\beta$-stimulated cytokine and chemokine secretion in vascular endothelial cells by AMP-activated protein kinasedependent and -independent mechanisms. Sci Rep 8(1):5276. https://doi.org/10.1038/s41598-018-23420-4

33. Hawley SA, Ford RJ, Smith BK et al (2016) The Na+/glucose cotransporter inhibitor canagliflozin activates AMPK by inhibiting mitochondrial function and increasing cellular AMP levels. Diabetes 65(9):2784-2794. https://doi.org/10.2337/db16-0058

34. Dailey MJ, Kim S (2012) Inositol polyphosphate multikinase: an emerging player for the central action of AMP-activated protein kinase. Biochem Biophys Res Commun 421(1):1-3. https://doi. org/10.1016/j.bbrc.2012.04.010

35. Cioffi F, Senese R, Lasala P et al (2017) Fructose-rich diet affects mitochondrial DNA damage and repair in rats. Nutrients 9(4):323. https://doi.org/10.3390/nu9040323

36. Scarpulla RC (2011) Metabolic control of mitochondrial biogenesis through the PGC-1 family regulatory network. Biochim Biophys Acta 1813(7):1269-1278. https://doi.org/10.1016/j.bbamc r.2010.09.019

37. Kondo Y, Masutomi H, Noda Y et al (2014) Senescence marker protein-30/superoxide dismutase 1 double knockout mice exhibit increased oxidative stress and hepatic steatosis. FEBS Open Bio 4:522-532. https://doi.org/10.1016/j.fob.2014.05.003

38. Kim YS, Gupta Vallur P et al (2017) Insights into the dichotomous regulation of SOD2 in cancer. Antioxidants 6(4):E86. https://doi. org/10.3390/antiox6040086

39. Yang Y, Wang J, Zhang Y, Li J, Sun W (2018) Black sesame seeds ethanol extract ameliorates hepatic lipid accumulation, oxidative stress, and insulin resistance in fructose-induced nonalcoholic fatty liver disease. J Agric Food Chem 66(40):10458-10469. https ://doi.org/10.1021/acs.jafc.8b04210

40. Fan X, Lv H, Wang L, Deng X, Ci X (2018) Isoorientin ameliorates APAP-induced hepatotoxicity via activation $\mathrm{Nrf} 2$ antioxidative pathway: the involvement of AMPK/Akt/GSK3 $\beta$. Front Pharmacol 9:1334. https://doi.org/10.3389/fphar.2018.01334

41. Hua X, Deng R, Li J, Chi W, Su Z, Lin J, Pflugfelder SC, Li DQ (2015) Protective effects of L-carnitine against oxidative injury by hyperosmolarity in human corneal epithelial cells. Investig Ophthalmol Vis Sci 56(9):5503-5511. https://doi.org/10.1167/ iovs. 14-16247

42. El-Sherbini ES, El-Sayed G, El Shotory R et al (2017) Ameliorative effects of L-carnitine on rats raised on a diet supplemented with lead acetate. Saudi J Biol Sci 24(6):1410-1417. https://doi. org/10.1016/j.sjbs.2016.08.010

43. Lee BJ, Lin JS, Lin YC, Lin PT (2014) Effects of L-carnitine supplementation on oxidative stress and antioxidant enzymes activities in patients with coronary artery disease: a randomized, placebo-controlled trial. Nutr J 13:79. https://doi. org/10.1186/1475-2891-13-79

44. Benedini S, Perseghin G, Terruzzi I et al (2009) Effect of L-acetylcarnitine on body composition in HIV-related lipodystrophy. Horm Metab Res 41(11):840-845. https://doi. org/10.1055/s-0029-1225625

Publisher's Note Springer Nature remains neutral with regard to jurisdictional claims in published maps and institutional affiliations. 